\title{
Validation by Scanning Electron Microscopy of Three-Dimensional Coating of PCL/PLLA Scaffolds with Electrospun PLLA Nanofibers for Tissue Engineering
}

\author{
Phillip E. McClellan and William J. Landis \\ University of Akron, Department of Polymer Science, Akron, OH, USA
}

Electrospinning is an efficient and cost effective method of generating nanofibers for use in filtration, sensors, wound healing, and tissue engineering. A key advantage in each of these applications results from the high surface area-to-mass ratio of the electrospun nonwoven nanofiber mats. With regard to tissue engineering, the specific orientation and composition of the nanofibers can be altered to resemble the three-dimensional structure of native extracellular matrix of many different tissues. For instance, aligned nanofibers can be collected on a rotating disk or drum and used to facilitate growth of nerve cells [1]. Typically, synthetic polymers such as polycaprolactone (PCL) and poly-L-lactic acid (PLLA) are utilized to form nanofibers although natural materials such as collagen and elastin with characteristics promoting cell attachment and proliferation may be electrospun as well [2]. Electrospun fibers are most often deposited onto flat surfaces of high electrical conductivity to facilitate even deposition and growth of nanofiber mats having relatively uniform thickness. Three-dimensional collectors such as metal cylinders have been utilized to form small tubes of nanofibers which may prove useful for engineering blood vessels [3].

Current techniques to create complex, three-dimensional materials using electrospun nanofibers have been limited and resulting electrospun materials often lack structural integrity required for high-load applications. A novel method to mitigate this issue has been devised by which deposition of fibers faithfully follows the surfaces of a material during the electrospinning process. The nanofibers adopt the shape of the specimen using such an approach and produce a thin, nanofiber "coating" over the surface of the object.

To establish the validity of the method proposed above, rectangular-shaped scaffolds composed of PCL/PLLA $(75 / 25)$ were coated with nanofibers of PLLA by utilizing this unique electrospinning technique. Briefly, each scaffold was mounted onto the tip of a fine-point, stainless steel needle connected to a grounded, flat metal electrode. A solution composed of PLLA in chloroform was loaded into a syringe having a blunt needle attached to it. Nanofibers of PLLA were electrospun to the surface of the scaffolds utilizing an applied voltage of $13.1 \mathrm{kV}$ and a working distance of $6 \mathrm{~cm}$ between the electrospinning tip and fine-point needle tip. Coated scaffolds were removed carefully from the stainless steel needle and transferred to a small petri dish and dried overnight at room temperature.

Dry, nanofiber-coated scaffolds were immersed in $100 \%$ ethanol for 24 hours for sterilization and to ensure the nanofibers remained adhered to the scaffolds in a liquid environment. The nanofiber-coated scaffolds were then removed from ethanol and dried over 24 hours. Representative samples were subsequently mounted onto copper stubs, sputtercoated with silver, and examined in a JEOL model 7401 scanning electron microscope operated at multiple accelerating voltages $(1.0-2.0 \mathrm{kV})$. Images were collected and recorded digitally and showed coverage of PLLA nanofibers over all six surfaces of the polymer scaffolds. Nanofibers accumulated in "peaks" about the points of needle protrusion from specimen surfaces (Figure 2A) and the specimen surface facing away and hidden from the ejected jet of electrospun nanofibers was also coated although with a lower percentage of total surface coverage 
(Figure 2B). Nanofiber coverage is increased by lengthening the time utilized to coat the specimen. The process of applying nanofiber coatings to complex three-dimensional objects is not limited to biodegradable materials for tissue engineering. It can be utilized in many applications requiring close and reliable reproduction of a nanofiber coating on non-conductive three-dimensional objects [4].

\section{References:}

[1] Yang F, et al. Biomaterials 26 (2005), p. 2603-2610.

[2] Buttafoco L, et al. Biomaterials 27 (2006), p. 724-734.

[3] Zhang D and Chang J. Nano Letters 8 (2008), p. 3283-3287.

[4] The authors thank Dr. Darrell Reneker (Department of Polymer Science, University of Akron) for providing laboratory access and Mr. Daniel Clark (Department of Polymer Science, University of Akron) for assisting in the initial setup of the basic electrospinning apparatus.

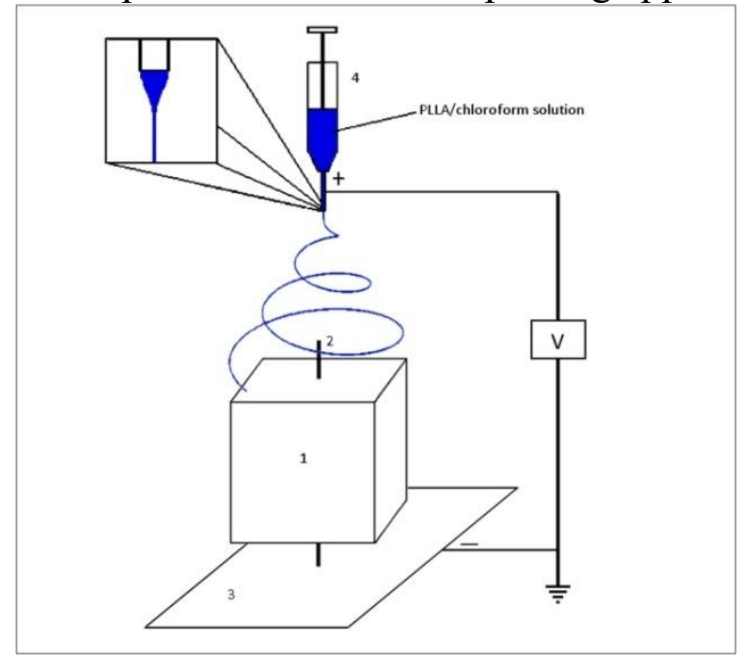

Figure 1. Schematic of the electrospinning design for applying nanofibers to a three-dimensional tissue scaffold. The PCL/PLLA scaffold (1) is mounted on a fine stainless steel needle (2) directly connected to a flat, grounded collector (3). A $1.0 \%$ PLLA/chloroform solution loaded into a syringe (4) is delivered by action of a syringe pump that controls the solution flow rate.

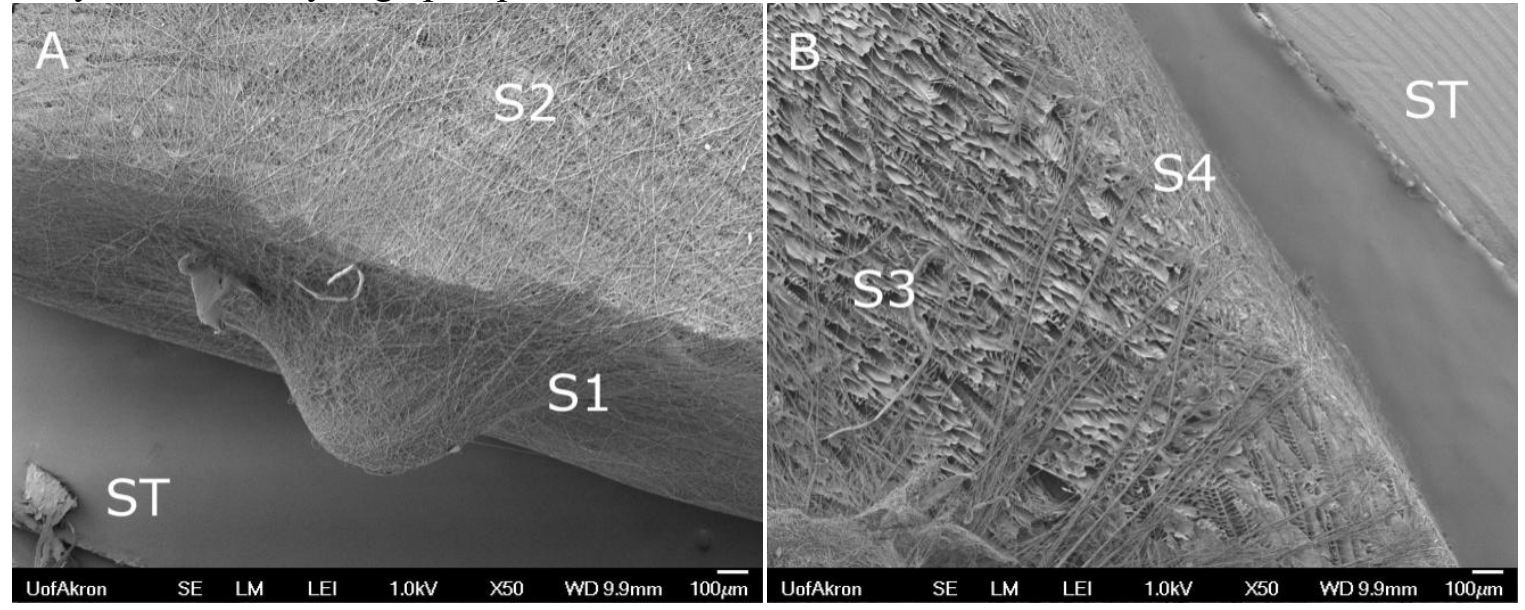

Figure 2. (A) Nanofibers coat surfaces (S1-S4) of PCL/PLLA specimens and collect in a "peak" at the point of needle protrusion. (B) Specimen surfaces (S3) completely hidden from the electrospinning tip are coated with nanofibers as well as adjoining surfaces (S4). The copper support stub is labeled (ST). 